\title{
Access and Accessibility for the London Mathematical Society Journals
}

Susan Hezlet

The title of this article, inspired by Sense and Sensibility, is set to highlight the distinction between what may be easily accessible, for example, freely available to the reader in some version or other, and what is accessed or downloaded and read from our journals' websites. At the London Mathematical Society (LMS) we have been gathering usage data on the full-text downloads from which we can see the papers that are read most frequently and if the presence of a preprint version on the arXiv has an effect on the usage of the final published version. In the UK we are undergoing a change in funders' policies that is driving a move towards paid open access. I will describe how the Society is responding to the government policy, with the aim of providing a balanced mixture of access policies that support both authors and readers.

Why should we be interested in usage data and the effect of the arXiv? We use the analysis to inform our decisions on what to make accessible through our publishing policies for the journals. We want to open up access as far as possible while not undermining the income that pays for the costs of production and supports the activities of the Society. The costs of production include the cost of managing the refereeing of papers, that is, providing staff to support the authors, referees, and editors from submission to decision and to reduce time spent waiting for referee reports where possible. Then there is the cost of copyediting, typesetting, and proofing, a service appreciated by the

Susan Hezlet is publisher at the London Mathematical Society. Her email address is susan. hezlet@1ms . ac.uk. Members of the Editorial Board for Scripta Manent are: Jon Borwein, Thierry Bouche, John Ewing, Andrew Odlyzko, and Ann Okerson.

DOI: http://dx.doi.org/10.1090/noti1085

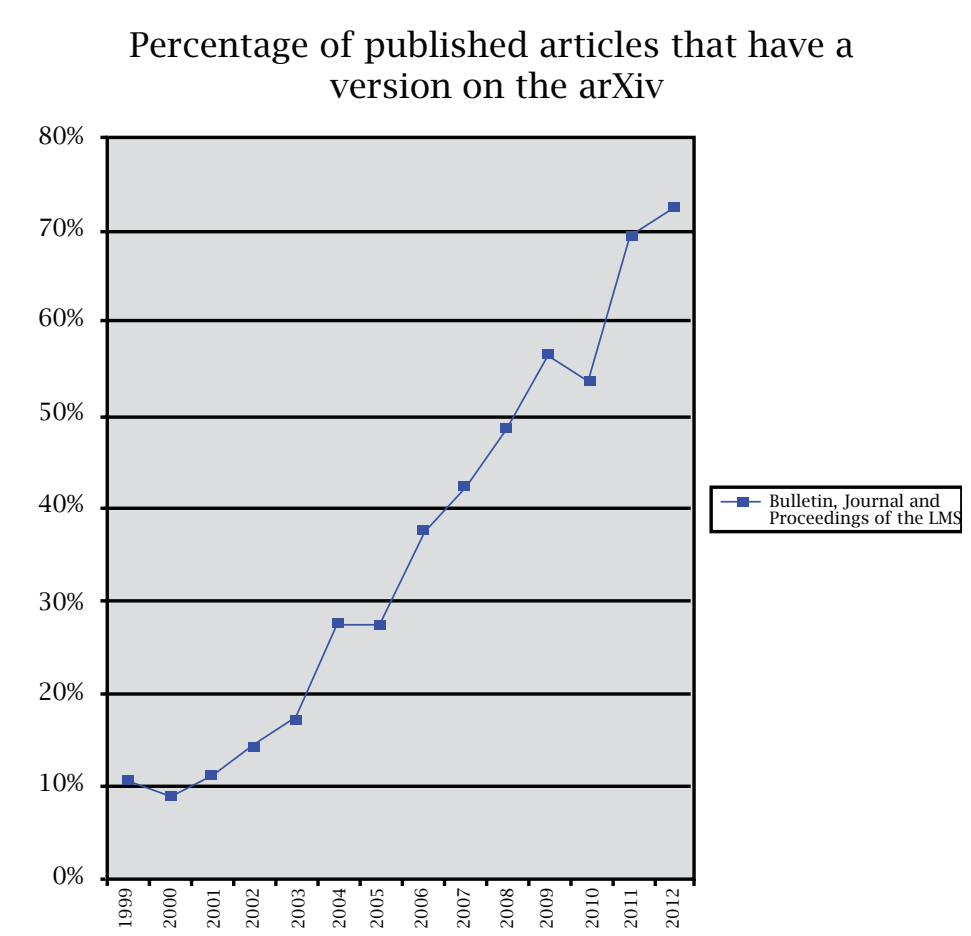

Graph 1. Percentage of published articles that have a version on the arXiv.

majority of our authors whose first language is not English. Another cost is the sustainable preservation of the older articles in a form that is regularly upgraded to take account of new developments in electronic media.

The obvious question for a publisher to ask is if the presence of the arXiv is a threat to published journals. At the LMS we have been tracking the growth in the percentage of our published papers that have a version on the arXiv for almost fifteen years, and the results are given in graph 1 . The 


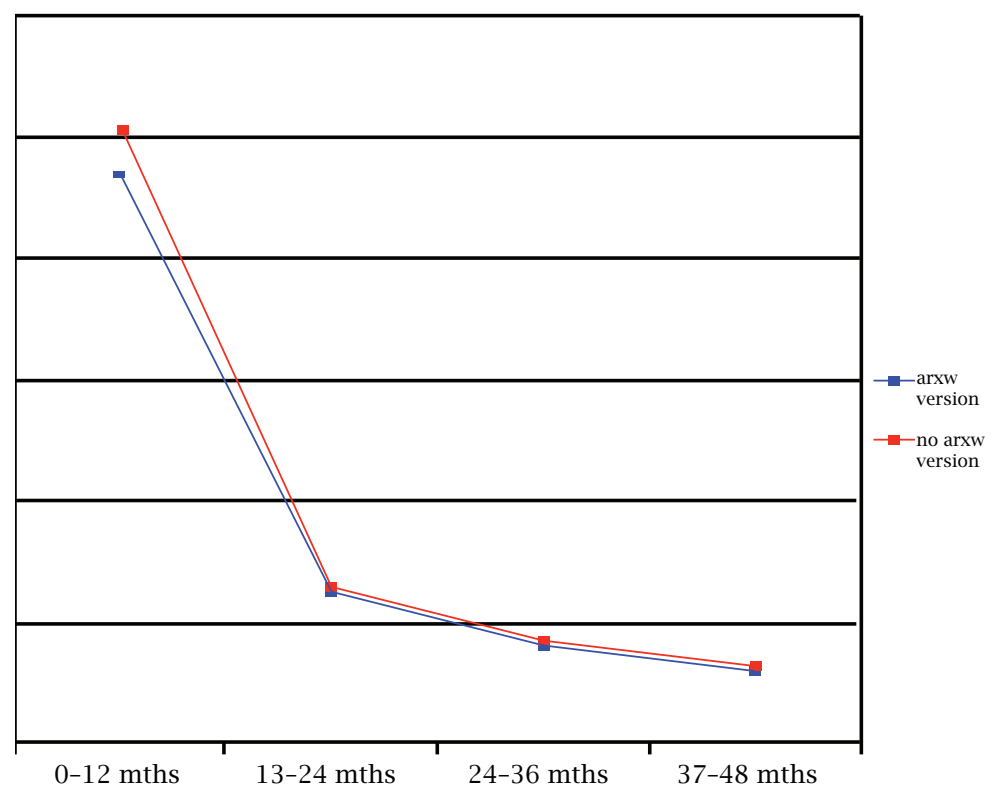

Graph 2. Average number of full text downloads per period, all papers, Bulletin, Journal, and Proceedings of the LMS.

Bulletin, Journal and Proceedings are general journals, and the average growth across all of mathematics is slower than for those subject areas whose mathematicians were "early adopters", such as the algebraic geometers.

There have been accounts from theoretical particle physics of a serious decline in readership of published journals where almost all of the articles are available in preprint form on the arXiv.

At the LMS we decided to see if we could find a similar result by identifying those papers published in the Bulletin, Journal and Proceedings that have an arXiv version and comparing that group of journal articles with the rest, i.e., those articles where there is no arXiv version. The results are shown in graph 2.

During the first twelve months of publication, ${ }^{1}$ there is a small and clear distinction between the two lines, showing that papers not on the arXiv (the red line) are downloaded more frequently on average than those that have a preprint version on the arXiv (the blue line). This effect quickly tails off in subsequent years, and over all the years we have measured, the tiny difference shown in graph 2 cannot be seen as a threat to our journals. This result came as a welcome surprise, and a similar pattern is seen for our other journals.

It might be true that, if the arXiv had never existed, published research articles in mathematics would be more widely read, but the arXiv is a reality and it is pointless to speculate on its nonexistence.

${ }^{1}$ The date of publication is the date on which the online version of the paper is published. The online version is now the final "version of record" and is not posted until after all production work is completed.
For me as a publisher, it is a reassuring confirmation that published papers are read regardless of their "pre-life" on the arXiv and there is a value to the long-term preservation and storage of the mathematical literature in an "organized" form, i.e., as journals, where the Society has a duty to look after the published record.

To give an idea of the scale of the data, we looked at 1,332 articles, of which 562 did not have a version on the arXiv. The search for each arXiv version was a painstaking manual job and there will be some human errors, but this works in both directions: misidentifying a preprint version through two papers having similar titles and failing to recognize the preprint version because there has been a substantial change in the title.

One of the reasons for the significantly higher number of downloads in the first twelve months of publication is that we give free access to all the papers in these journals for the first six months (this is called a "reverse moving wall"). This policy has been in place for ten years now, and there is no evidence to suggest it affects a library's decision to subscribe to the journal. I also looked at usage data for two journals that do not offer the reverse moving wall, and while there is higher usage in the first year of publication, which then tails off in subsequent years, it is only about half of the usage we see in graph 2.

The reason we adopted the reverse moving wall is because we wanted to encourage early reading and citing of the papers while, at the same time, recognizing the value of the older papers. Mathematics truly is a long-lived subject. All of our journals that have been published for more than twenty years have cited half-lives greater than ten years. $^{2}$ In 2012 the Proceedings of the LMS had a cited half-life of thirty-three years. We also have preliminary data that the usage half-life of the Proceedings is over ten years. For both the usage and the citation data, it appears that several famous old papers are dominant, for example, those by Maxwell, Kelvin, Turing, and Hodge.

However, we may have to close early access in the face of being required to make other parts of the journal freely available under new open access policies driven by changes in UK government policy. Over the last year we have had to review our publishing policies across all our journals following the decision of the UK Department of Business and Industry to adopt a radical policy for publicly funded research, summarized in the flowchart on the next page.

\footnotetext{
${ }^{2}$ The cited half-life is measured by taking all the citations made in a single year (e.g., 2012) to any papers published in a journal in any year of publication dating back to the journal's first volume (e.g 1865 for the Proceedings.) The "half-life" year is the year where the median point is found.
} 


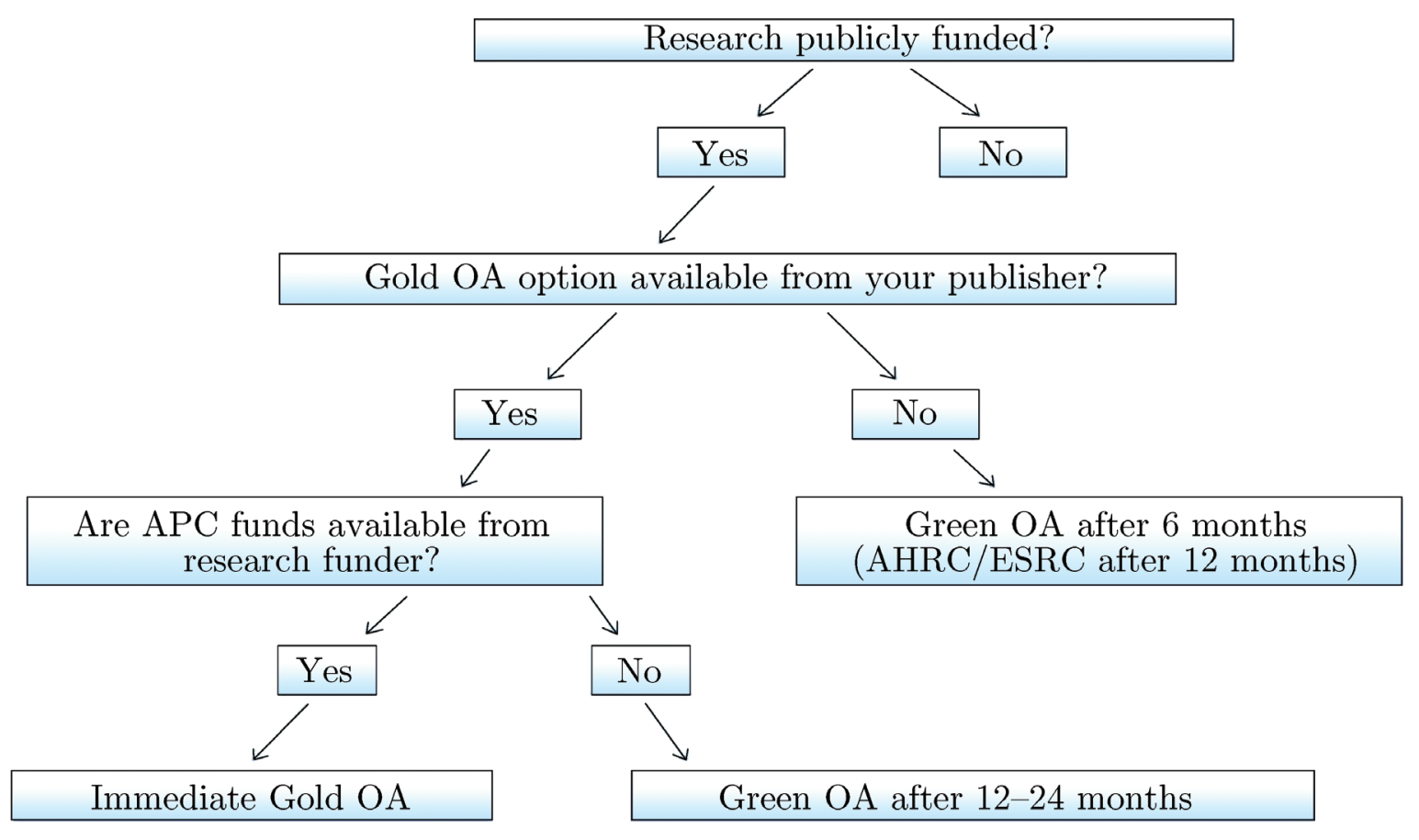

I won't go into the details of this chart, the full explanation for which can be found on the Research Council's UK website. ${ }^{3}$ My intention in showing it is to give an idea of the increasingly complex world in which mathematical researchers are finding themselves, particularly where there are coauthors from several countries, some of whom may be working under conflicting policies.

The Society has been active in defending the uniqueness of mathematics research and its expression through published journals, and while government statements supported the idea that special cases could be made, recognizing that "one size does not fit all," nothing has been done in practice. Mathematics as just one of the subjects covered by the UK Engineering and Physical Sciences Research Council (EPSRC) has to follow the same conditions and embargo periods as any other subject within this wide group, regardless of the presence of the arXiv or unique features such as long citation and usage half-lives.

Although we are a British-based society, more of our authors are from the USA than the UK; last year we had authors from forty-four countries contributing to the 280 articles published in the LMS journals. This is the point at which we set aside the question of "is open access a good or bad thing?" Instead we ask, "have we provided all the options to ensure that a mathematician from any country can submit a paper to our journals and find a place that fits his or her funder's requirements while retaining our high standards for acceptance

3 http://www. rcuk.ac.uk/research/Pages/outputs. aspx of a paper regardless of funding?" We have to balance the authors' needs with accessibility for our readers, ensuring their libraries get value for their subscriptions. Where there is no money for subscriptions, free access is subsidized through our developing countries initiative, and the presence of preprint versions on the arXiv provides a sustainable "green" alternative access.

We recognize that, for green open access, some people would like the final published version (the "version of record") to be freely available. Setting an embargo period to match the citation half-life of our journals would be unacceptable to some, but six or twelve months embargo periods are far too short, and there is a danger that such a policy would quickly lead to cancellations of subscriptions. We have evidence for this from the case of the Annals of Mathematics, which lost over one third of its subscriptions in five years when it moved to a green open access model. The distinguishing feature for the Annals was that it was the final published versions on the journal's website that were freely available, leading librarians to question paying subscriptions for online access to something freely available. From the scant evidence we have, it seems that the danger does not lie in the arXiv version because these are preprints and "disorganized" in comparison with hosting the free versions on the publisher's own website. I believe there would be a threat to the subscription base if we were required to deposit the final published version and not just the author's accepted manuscript. I should be clear that no one is asking this of our published papers; I have seen several summaries describing the development of 


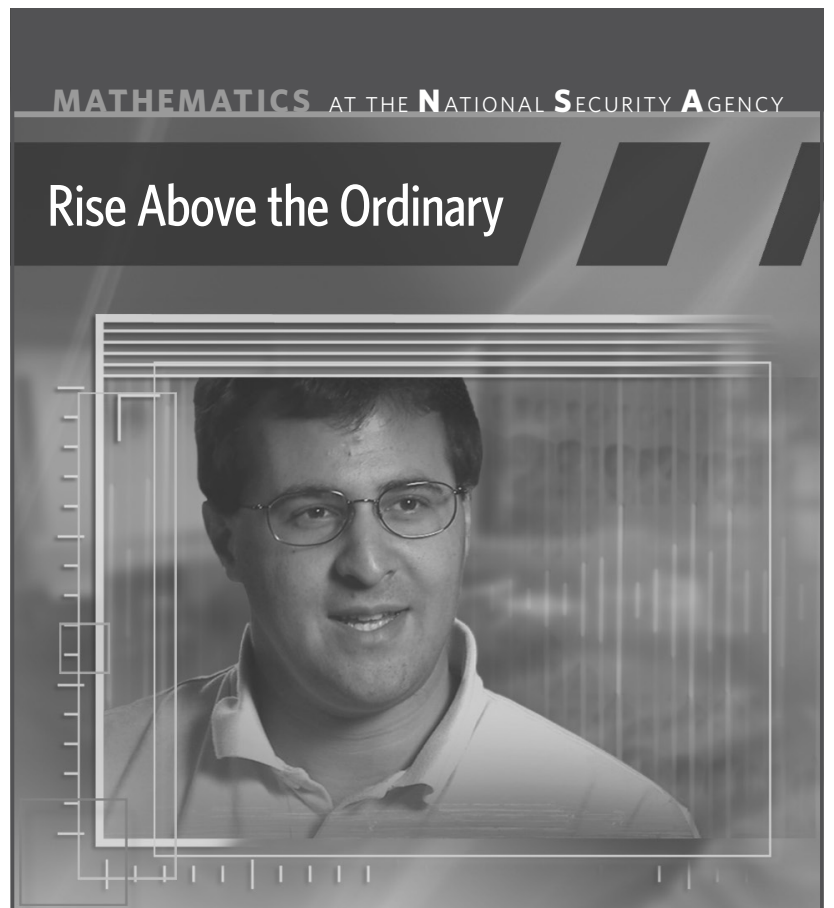

A career at NSA is no ordinary job. It's a profession dedicated to identifying and defending against threats to our nation. It's a dynamic career filled with challenging and highly rewarding work that you can't do anywhere else but NSA.

You, too, can rise above the ordinary. Whether it's producing valuable foreign intelligence or preventing foreign adversaries from accessing sensitive or classified national security information, you can help protect the nation by putting your intelligence to work.

NSA offers a variety of career fields, paid internships, co-op and scholarship opportunities.

Learn more about NSA and how your career can make a difference for us all.

\section{KNOWINGMATTERS}

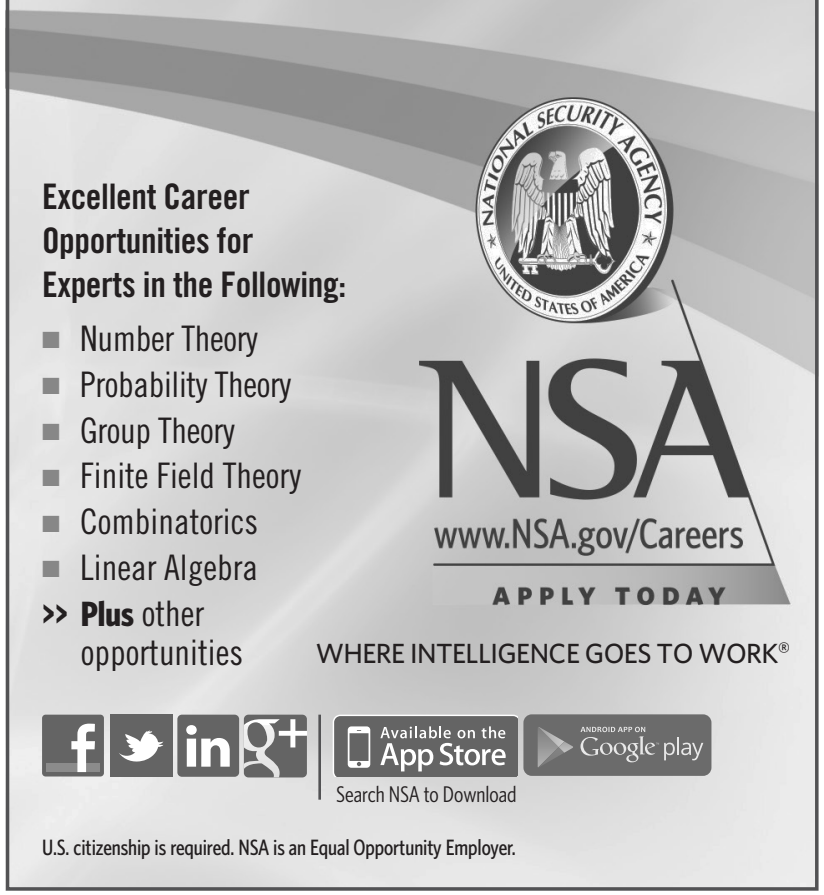

government polices around the world, and so far they only require deposit of the author manuscript after an embargo period. However, the policies are frequently badly worded and imprecise-and changing all the time!

Our response to this situation is to develop the following policies, which we hope cover all the bases for both readers and authors. There are three main options that apply to our core journals (the Bulletin, Journal, and Proceedings, and now the Transactions). We hope these will cover everything a mathematician may be required to do.

1) If an author would like to make his article permanently available in final published form, he can apply to his funder or university for the funds to pay the hybrid open access fee of the Bulletin, Journal, or Proceedings. Mathematicians who are awarded grants from EPSRC are first expected to follow this route. If there is no money left for the payment of the Author Payment Charges (APCs), then the authors may take the green route. Since the introduction of the UK policy, we have seen several universities (for example, Imperial College and Warwick) paying the hybrid fees.

2) A few funders have said they will not pay fees for hybrid journals, preferring only to fund pure open access journals. As a result, we have launched a new journal, the Transactions of the LMS, which is a gold open access journal. Note that this is not a "transfer journal" and the editor will not consider papers rejected by other LMS journals. We have several papers currently under review and expect to publish the first papers this year.

For further information on the open access options and the launch of the Transactions, see http://www.1ms.ac.uk/sites/7ms.ac.uk/ files/Publications/TLMS-Announcement. pdf, which explains the background of the decision to launch the journal and how it fits in with our other journals.

Our Editorial Advisory Board now looks after papers submitted to the Bulletin, Journal, Proceedings, and Transactions and ensures that the same high standards are applied to all four journals.

3) Authors in any of these journals are free to post their preprint versions, up to the final accepted version, on the arXiv and in any repository that they are required to do so. This has been the case for many years.

In this article I have described in some detail what the LMS is doing about open access, and I think have fairly reflected the reasons why we have adopted these policies and gone so far as to launch a new open access journal. However, the views expressed here are my own and shouldn't be taken to represent the membership or the Council of the LMS. After all, with over two thousand members, the range of their views should cover every possible opinion on publishing. 